\title{
Anatomical image-guided fluorescence molecular tomography reconstruction using kernel method
}

Reheman Baikejiang

Yue Zhao

Brett Z. Fite

Katherine W. Ferrara

Changqing Li 


\title{
Anatomical image-guided fluorescence molecular tomography reconstruction using kernel method
}

\author{
Reheman Baikejiang, ${ }^{a}$ Yue Zhao, ${ }^{a}$ Brett Z. Fite, ${ }^{b}$ Katherine W. Ferrara, ${ }^{b}$ and Changqing Li ${ }^{a, *}$ \\ aUniversity of California, Merced, School of Engineering, Merced, California, United States \\ bUniversity of California, Davis, Department of Biomedical Engineering, Davis, California, United States
}

\begin{abstract}
Fluorescence molecular tomography (FMT) is an important in vivo imaging modality to visualize physiological and pathological processes in small animals. However, FMT reconstruction is ill-posed and ill-conditioned due to strong optical scattering in deep tissues, which results in poor spatial resolution. It is well known that FMT image quality can be improved substantially by applying the structural guidance in the FMT reconstruction. An approach to introducing anatomical information into the FMT reconstruction is presented using the kernel method. In contrast to conventional methods that incorporate anatomical information with a Laplacian-type regularization matrix, the proposed method introduces the anatomical guidance into the projection model of FMT. The primary advantage of the proposed method is that it does not require segmentation of targets in the anatomical images. Numerical simulations and phantom experiments have been performed to demonstrate the proposed approach's feasibility. Numerical simulation results indicate that the proposed kernel method can separate two FMT targets with an edge-to-edge distance of $1 \mathrm{~mm}$ and is robust to false-positive guidance and inhomogeneity in the anatomical image. For the phantom experiments with two FMT targets, the kernel method has reconstructed both targets successfully, which further validates the proposed kernel method. (C) 2017 Society of Photo-Optical Instrumentation Engineers (SPIE) [DOI: 10.1117/1.JBO.22.5.055001]
\end{abstract}

Keywords: fluorescence molecular tomography; anatomical guidance; kernel method.

Paper 170015R received Jan. 5, 2017; accepted for publication Apr. 10, 2017; published online May 2, 2017.

\section{Introduction}

Fluorescence molecular tomography (FMT) has been emerging as an optical imaging modality for many years. FMT, as an important molecular imaging tool, has a broad range of applications in biomedical studies from drug development in small animal models ${ }^{1-8}$ to the clinical diagnosis in humans. ${ }^{9-11}$ However, due to the strong scattering nature of optical photons in deep tissues and a limited number of measurements, the inverse problem of FMT is ill-posed and underdetermined, which results in low spatial resolution in FMT imaging, in particular for targets in deep turbid media.

Many approaches have been proposed to improve the FMT image quality, including multispectral wavelengths for both excitation and emission wavelengths, different illumination patterns, ${ }^{12,13}$ a large number of FMT measurements by using charge-coupled device cameras, ${ }^{14-18}$ and improved FMT reconstruction algorithms-especially the sparse enhancement FMT reconstruction for the sparse FMT targets. ${ }^{19-24}$ A region reconstruction method implemented with level set method was also introduced to improve the FMT image reconstruction. ${ }^{25,26}$ A thorough review of FMT imaging in terms of instruments, methods, and applications was presented in Ref. 27.

Although numerous efforts have been implemented to improve FMT, its spatial resolution is still worse than those of other functional imaging modalities, such as functional magnetic resonance imaging, single-photon emission computed tomography, and positron emission tomography (PET). To further improve the spatial resolution of FMT, structural guidance from other anatomical images has been introduced into the FMT. ${ }^{4,28-30}$ Davis et al. ${ }^{3,5,10,28,31}$ reported the magnetic resonance imaging (MRI)-coupled FMT implemented with the Laplaciantype regularization. Schulz et al. ${ }^{7,8,29,32}$ reported a hybrid system for simultaneous FMT and x-ray computed tomography; Stuker et al. $^{33}$ reported the combined MRI and FMT system using single photon avalanche diode detectors. Recently, microscopic positron emission tomography (micro-PET), with a spatial resolution up to $1 \mathrm{~mm}$, has been used to guide FMT imaging. ${ }^{30,34}$ More recently, trimodality ${ }^{35}$ and even pentamodality, tomographic imaging systems were also investigated. ${ }^{36}$

One of major challenges in the multimodality FMT system is how to utilize anatomical information properly and easily in the FMT reconstruction. Soft prior method is a widely accepted approach, which allows variations within the segmented regions. Local Laplace and weighted segments have also been introduced to FMT reconstruction. ${ }^{29,32}$ It has been demonstrated that the combination of Laplace with weighted segments performed best in terms of quantification and localization. However, both methods require image segmentation, which is time consuming and prone to human error. To eliminate the need for prior image segmentation, Holt et al. ${ }^{37}$ reported a direct regularization method, in which the anatomical image grayscale values were introduced into a regularization operator. Similarly, our proposed kernel method also eliminates the need of anatomical image segmentation. The major difference is that our approach does not need the regularization operator, which allows us to have maximum flexibility to implement this method. 
In this paper, inspired by the kernel method in PET image reconstruction, ${ }^{38}$ we introduce the kernel-based image reconstruction as an approach to incorporating anatomical guidance into FMT. Compared with the Laplacian-type regularization methods, the proposed kernel method does not require the target region segmentation. Furthermore, as demonstrated by the numerical simulations in this paper, the proposed kernel method is robust to the false-positive guidance and inhomogeneity in the anatomical images.

In the kernel method, the fluorophore concentration at a node $i$ is defined as a function of a set of features, $\mathbf{f}_{i}$, which is directly extracted from the voxel intensities of the corresponding anatomical three-dimensional (3-D) images. Then, the kernelized FMT image model is incorporated into the forward model of FMT. Due to the simplicity of this model, we can combine it with any FMT reconstruction algorithm. In this study, we used a kernelized projection model with the majorization-minimization (MM) approach. ${ }^{39,40}$

The rest of this paper is organized as follows: in Sec. 2, we describe the FMT forward model, the regularized reconstruction method of FMT, and the proposed kernel-based reconstruction algorithm. In Sec. 3, numerical simulations and experimental results are presented. Finally, we conclude the paper with discussions in Sec. 4.

\section{Methods}

\subsection{Forward Model and Reconstruction Algorithms of FMT}

Light propagation in tissues is dominated by optical scattering and can be modeled by the diffusion equation. ${ }^{41}$ For FMT in the continuous wave domain, the light propagation model in 3-D is described by a set of coupled differential equations that are given below ${ }^{42,43}$

$$
\left\{\begin{aligned}
-\nabla \cdot\left[D_{e x}(\mathbf{r}) \nabla \Phi_{e x}(\mathbf{r})\right]+\mu_{\alpha, e x} \Phi_{e x}(\mathbf{r}) & =\delta_{s}\left(\mathbf{r}-\mathbf{r}_{s}\right) \\
\mathbf{n} \cdot\left[D_{e x}(\mathbf{r}) \nabla \Phi_{e x}(\mathbf{r})\right]+\alpha_{e x} \Phi_{e x}(\mathbf{r}) & =0 \\
-\nabla \cdot\left[D_{e m}(\mathbf{r}) \nabla \Phi_{e m}(\mathbf{r})\right]+\mu_{\alpha, e m} \Phi_{e m}(\mathbf{r}) & =\Phi_{e x}(\mathbf{r}) \mathbf{x}(\mathbf{r}) \\
\mathbf{n} \cdot\left[D_{e m}(\mathbf{r}) \nabla \Phi_{e m}(\mathbf{r})\right]+\alpha_{e m} \Phi_{e m}(\mathbf{r}) & =0
\end{aligned}\right.
$$

where $\nabla$ denotes the gradient operator, $D(\mathbf{r})=$ $\left\{3\left[\mu_{s}^{\prime}(\mathbf{r})+\mu_{a}(\mathbf{r})\right]\right\}^{-1}$ is the diffusion coefficient, $\mu_{a}(\mathbf{r})$ is the absorption coefficient, $\mu_{s}^{\prime}(\mathbf{r})$ is the reduced scattering coefficient, $\Phi(\mathbf{r})$ is the photon fluence at the location $\mathbf{r}, \delta_{s}\left(\mathbf{r}-\mathbf{r}_{s}\right)$ is Dirac delta function defining point source, $\mathbf{x}$ is the unknown to be reconstructed, which is related to the fluorescent dye concentration and the quantum yield at each node, ${ }^{23} \mathbf{n}$ is the outward unit normal vector of the boundary, and $\alpha$ is the Robin boundary coefficient. In Eq. (1), subscripts of $e x$ and $e m$ mean corresponding terms at the excitation and emission wavelengths, respectively. Equation (1) can be solved by the finite element method based on a finite element mesh and is linearized to the following equation:

$K_{e x} \Phi_{e x}=\delta_{s}\left(\mathbf{r}-\mathbf{r}_{s}\right), \quad K_{e m} \Phi_{e m}=\Phi_{e x} \mathbf{x}$,

where $K_{e x}$ and $K_{e m}$ are the stiffness matrices at the excitation and emission wavelengths, respectively. With the conjugate gradient approach, ${ }^{16}$ the above equations can be described as ${ }^{42}$
$\mathbf{A x}=\mathbf{b}$,

where $\mathbf{A} \in \mathbf{R}^{\left\{N_{m} \times N_{n}\right\}}$ is the system matrix, $\mathbf{x} \in \mathbf{R}^{\left\{N_{n} \times 1\right\}}$ is the unknown related to the fluorophore distribution or the FMT image to be reconstructed, $\mathbf{b} \in \mathbf{R}^{\left\{N_{m} \times 1\right\}}$ is the measurement vector, $N_{n}$ is the finite element node number, and $N_{m}$ is the number of measurements.

Because of the ill-conditioned and ill-posed nature, Eq. (3) is usually solved as a regularized least square problem with the nonnegativity constraint

$\mathbf{x}=\underset{\mathbf{x}, \mathbf{x} \geq 0}{\operatorname{argmin}} \Phi(\mathbf{x})=:\|\mathbf{A} \mathbf{x}-\mathbf{b}\|_{2}^{2}+\lambda\|\mathbf{x}\|_{1}$,

where $\lambda$ is $L_{1}$ the regularization parameter.

In this study, for the case without anatomical guidance, Eq. (4) is solved by the MM approach that updates the FMT image iteratively to minimize the mismatch between the model predictions and the measurements. ${ }^{39,40,43}$

\subsection{Soft Prior Method}

When structured priors are present, the objective function of the FMT with Laplacian regularization will be

$\mathbf{x}=\underset{\mathbf{x}, \mathbf{x} \geq 0}{\operatorname{argmin}} \Phi(\mathbf{x})=:\|\mathbf{A x}-\mathbf{b}\|_{2}^{2}+\lambda\|\mathbf{L x}\|_{2}^{2}$.

In soft prior method, regularization matrix $\mathbf{L}$ is defined as ${ }^{10}$

$\mathbf{L}_{i j}= \begin{cases}1, & \text { for } i=j \\ -\frac{1}{N}, & \text { if } i \text { and } j \text { are in the same region, } \\ 0, & \text { otherwise }\end{cases}$

where $N$ is the number of nodes in that region. In Eq. (5), regularization term $\|\mathbf{L x}\|_{2}^{2}$ can be treated as a special case of $\|\mathbf{A x}-\mathbf{b}\|_{2}^{2}$ when $\mathbf{b}=0$. Then, it can be solved by the MM approach as described in Refs. 39, 40, and 43.

\subsection{Kernel-Based Anatomically Aided Reconstruction Algorithm}

The anatomically aided FMT reconstruction algorithms usually incorporate the anatomical guidance as a regularization matrix, which enhances the smoothness within the anatomical regions and also allows sharp transition between the different regions. ${ }^{37}$ In this paper, we introduce the kernel method, which includes the anatomical guidance into the projection model of FMT. The fluorophore distribution at the node $i$ is defined with a kernel function as s $^{38,44,45}$

$\mathrm{x}_{i}=\sum_{j} \alpha_{j} \kappa\left(\mathbf{f}_{i}, \mathbf{f}_{j}\right)$,

where $\mathbf{f}_{i}$ and $\mathbf{f}_{j}$ are the anatomical feature vectors corresponding to the finite element nodes of $i$ and $j$, respectively. These anatomical feature vectors are directly extracted from the corresponding voxels in the 3-D anatomical images for each finite element node. The finite element mesh and the anatomical images should be coregistered. In some reported multimodality FMT systems, accurate coregistrations were reported, ${ }^{7,33}$ which makes the proposed kernel method to be implemented easily. It is also worth pointing out that voxels corresponding to finite 
element nodes on the surface of the mesh and outside of the mesh are excluded from the feature vector extraction. The length of the feature vectors depends on the voxels number. For example, for a voxel number of $3 \times 3 \times 3$, the length of the feature vector is 27 .

In Eq. (7), $\kappa$ is the kernel function. There are a variety of choices of the kernel function $\kappa^{46,47}$ Here, we use the radial Gaussian kernel ${ }^{48}$

$\kappa\left(\mathbf{f}_{i}, \mathbf{f}_{j}\right)=\exp \left(\frac{-\left\|\mathbf{f}_{i}-\mathbf{f}_{j}\right\|^{2}}{\sigma^{2}}\right)$,

where the parameter $\sigma$ controls the edge sensitivity and yields more accurate results when $\sigma=1 .{ }^{49}$ For computational efficiency, a $k$-nearest neighbor (knn) search is carried out for each feature vector corresponding to each finite element node using the "knnsearch" function in MATLAB. The search is carried out according to the Euclidean distance between the feature vectors, not the physical distance between the finite element nodes in the Cartesian coordinate. Only those elements corresponding to the $k$-nearest neighbors are stored in the kernel matrix, and the rest of them are set to be 0 . This will result in the following definition of the kernel matrix:

$K_{i j}=\left\{\begin{array}{ll}\kappa\left(\mathbf{f}_{i}, \mathbf{f}_{j}\right), & \mathbf{f}_{j} \in \text { knn of } \mathbf{f}_{i} \\ 0, & \text { otherwise }\end{array}\right.$.

Thus, the kernel matrix is a sparse symmetric $N_{n} \times N_{n}$ matrix. The kernel matrix is normalized in this study for higher image quality ${ }^{38}$

$$
\overline{\mathbf{K}}=\operatorname{diag}^{-1}\left[\mathbf{K} \mathbf{1}_{N}\right] \mathbf{K}
$$

where $\mathbf{1}_{N}$ is a vector of all ones. Equation (7) can be written in a matrix-vector form as

$\mathbf{x}=\overline{\mathbf{K}} \boldsymbol{\alpha}$,

where the vector $\boldsymbol{\alpha}$ is a new unknown vector referred as the coefficient image. By substituting Eq. (11) into Eq. (3), the kernelized projection model of FMT can be written as

$\mathbf{A} \overline{\mathbf{K}} \boldsymbol{\alpha}=\mathbf{b}$.
Combining the kernelized projection model of Eq. (12) with the objective function of Eq. (4) leads to the following objective function:

$\boldsymbol{\alpha}=\operatorname{argmin} \Phi(\boldsymbol{\alpha})=:\|\mathbf{A} \overline{\mathbf{K}} \boldsymbol{\alpha}-\mathbf{b}\|_{2}^{2}+\lambda\|\boldsymbol{\alpha}\|_{1}$.

Because the reconstructed images are already regularized by the kernels, we set the regularization parameter in Eq. (13) to zero in this study ${ }^{38}$ and solved it by the MM approach. ${ }^{39,40}$ Once $\boldsymbol{\alpha}$ is obtained, we can easily obtain the final fluorophore distribution image by the linear transformation $\mathbf{x}=\overline{\mathbf{K}} \boldsymbol{\alpha}$.

\subsection{Numerical Simulation Setup}

\subsubsection{Cylindrical simulation phantom}

In this simulation, we used a cylindrical phantom with a diameter of $22 \mathrm{~mm}$ and a height of $80 \mathrm{~mm}$. Cylindrical targets with a diameter of $1.4 \mathrm{~mm}$ and a length of $20 \mathrm{~mm}$ were embedded $20 \mathrm{~mm}$ below the top surface of the phantom. In the coordinate system, the base of the cylinder was a circle on the $x-y$ plane centering the origin of the coordinate system and the height was along the $z$ axis. In this simulation, two targets were embedded at $(-1.7$ and 5.56) and (1.7 and 5.56) in the $x-y$ plane with an edge-to-edge distance of $2 \mathrm{~mm}$ as shown in Fig. 1(a).

In this and following numerical simulations, the phantom tissue optical properties were set to be $\mu_{a}=0.012 \mathrm{~mm}^{-1}$ and $\mu_{s}^{\prime}=$ $0.83 \mathrm{~mm}^{-1}$ at both the excitation wavelength $(650 \mathrm{~nm})$ and the emission wavelength $(700 \mathrm{~nm})$. We assigned the fluorophore concentration to be 1 in the target regions and 0 in the background regions.

The numerical phantom was discretized with a 3-D tetrahedral finite element mesh with 29,989 nodes and 155,310 elements. Numerical FMT measurement data were generated by Eq. (3) with a line pattern laser projected on the phantom surface. The line laser had a width of $1 \mathrm{~mm}$ and a length of $50 \mathrm{~mm}$. We had 30 excitation positions of the line laser to cover the whole surface. ${ }^{50}$ For each line laser excitation, the 9280 surface nodes on the side of the cylinder were used as the measurement detectors. Then, we added $30 \%$ Gaussian noise to the numerical FMT measurement data.

The 3 -D CT images with $220 \times 220 \times 801$ voxels were generated with a grid size of $0.1 \mathrm{~mm}$. The intensities of target
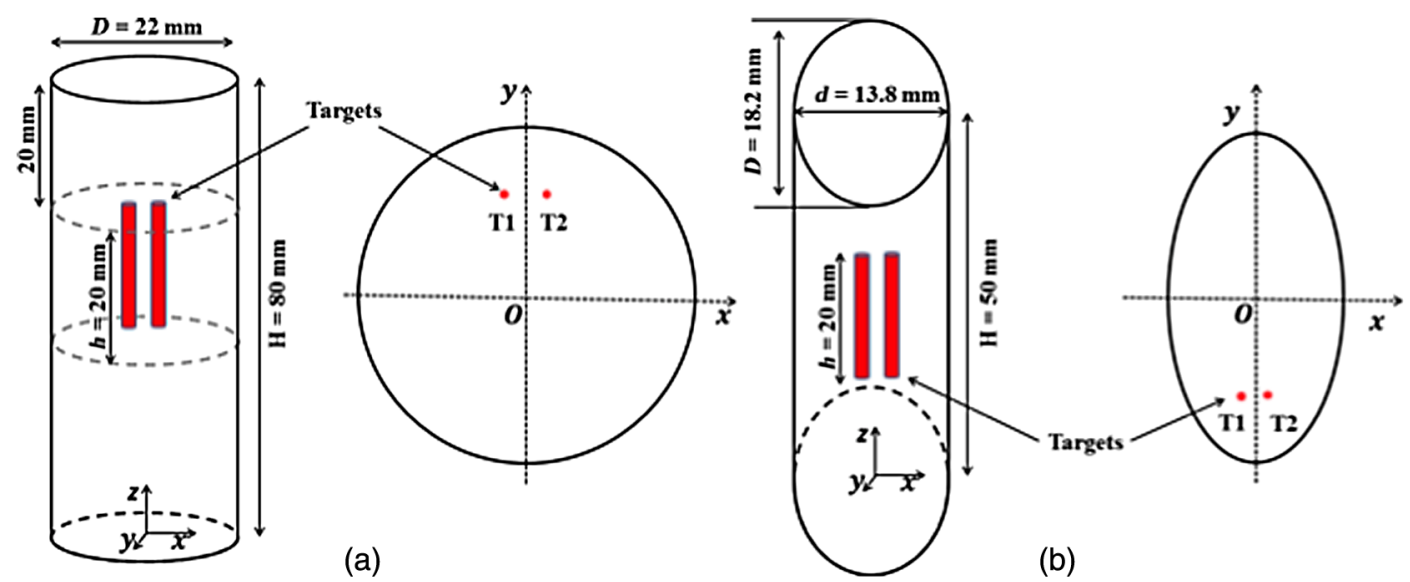

Fig. 1 Numerical simulation phantom geometry of (a) the cylindrical phantom with target locations at T1 $(-1.7$ and 5.56) and T2 (1.7 and 5.56) and (b) the elliptic cylindrical phantom with target locations at T1 $(-1.2$ and -5.0$)$ and T2 (1.2 and -5.0$)$. 
regions and the background were set to be 0.24 and 0.06 , respectively, which were close to the CT data in the phantom experiments. We added $15 \%$ Gaussian noise to the numerical CT images.

\subsubsection{Elliptic cylindrical simulation phantom}

In this simulation, we used an elliptic cylindrical phantom with a horizontal semiaxis of $6.9 \mathrm{~mm}$, vertical semiaxis of $9.2 \mathrm{~mm}$ and a height of $50 \mathrm{~mm}$. Two cylindrical targets with a diameter of $1.4 \mathrm{~mm}$ and a length of $20 \mathrm{~mm}$ were embedded $20 \mathrm{~mm}$ below the top surface of the phantom. In the coordinate system, the base of the cylinder was an ellipse on the $x-y$ plane centering the origin of the coordinate system and the height was along the $z$-axis. Two targets were embedded at $(-1.2$ and -5.0$)$ and $(1.2$ and $-5.0)$ in the $x-y$ plane with an edge-to-edge distance of $1 \mathrm{~mm}$ as shown in Fig. 1(b). The numerical phantom was discretized with a 3-D tetrahedral finite element mesh with 32,882 nodes and 191,359 elements. Numerical FMT measurement data were generated by Eq. (3) with a line pattern laser projected on the phantom surface. The line laser had a width of $1 \mathrm{~mm}$ and a length of $50 \mathrm{~mm}$. We had 30 excitation positions of the line laser to cover the whole surface. ${ }^{50}$ For each line laser excitation, the 6013 surface nodes on the side surface of the cylinder were used as the measurement detectors. Then, we added $30 \%$ Gaussian noise to the numerical FMT measurement data.

The transverse sections of the CT images were generated using the "phantom" command in MATLAB [as shown in Fig. 5(b) in Sec. 3]. The region outside of the ellipse was trimmed. Then, we stacked the phantom images to generate 3 -D CT images with $234 \times 176 \times 501$ voxels. Two targets with a diameter of $1.4 \mathrm{~mm}$ and a length of $20 \mathrm{~mm}$ were added in the 3-D CT phantom. The intensity of the targets is 0.99 , which is $1 \%$ less than the intensity of edges of the ellipse. This is because the tumor has the contrast as high as bones in $\mathrm{CT}$ images with CT contrast agent injection. ${ }^{29}$ As in the first simulation, we added $15 \%$ Gaussian noise to the numerical CT images.

\subsubsection{Numerical simulation using MRI images of a rat brain}

The ultimate goal of the proposed kernel method is its application in anatomical image (such as CT or MRI) guided FMT for small animal studies. To validate the feasibility of the proposed method using in vivo anatomical guidance with heterogeneous structures, we used the MRI images of a rat brain as the anatomical guidance. MRI imaging was performed with a Bruker Biospec 7 Tesla (7T) small-animal scanner (Bruker BioSpin MRI, Ettlingen, Germany). A 72-mm internal diameter linear resonator was used for radio frequency transmission, and a fourchannel rat brain phased array surface coil was used for signal reception. The rat brain was imaged coronally with a fast-spinecho sequence (RARE; axial: TE/TR $=8 \mathrm{~ms} / 750 \mathrm{~ms}$; FOV = $40 \times 40 \mathrm{~mm}^{2} ; \quad$ MTX $=256 \times 256 ; \quad$ ST $/ \mathrm{SI}=1 \mathrm{~mm} / 1 \mathrm{~mm} ;$ $\mathrm{ETL}=4)$. Data were acquired and reconstructed using ParaVision 5.1 software (Bruker BioSpin MRI). The experiment was conducted under a protocol approved by the University of California, Davis, Animal Use and Care Committee (Davis, California). A male athymic nude rat, purchased from Harlan Laboratories (Hayward, California), was inoculated with $3 \times 10 \mathrm{~mm}^{6}$ U87 MG cells/10 $\mu \mathrm{L}$ intracranially. The rat was administered $0.5 \mathrm{mmol} / \mathrm{kg}$ of the small molecule gadolinium chelate, gadoteridol (bracco imaging) via bolus i.v. injection prior to $\mathrm{T} 1 \mathrm{w}$ imaging.

From the MRI images, we used the open-source software, "iso2mesh," to generated a 3-D finite element mesh with 181,686 tetrahedral elements and 41,427 nodes. ${ }^{51}$ We segmented the tumor in the MRI images as the FMT target region. Similar to the numerical phantom studies, numerical FMT measurement data were generated by Eq. (3) with a line pattern laser projected on the rat brain surface. We had 30 excitation positions of the line laser to cover the whole rat brain surface..$^{50}$ For each line laser excitation, the 20,055 surface nodes were used as the measurement detectors. The tissue optical properties were set to $\mu_{a}=0.012 \mathrm{~mm}^{-1}$ and $\mu_{s}^{\prime}=0.83 \mathrm{~mm}^{-1}$ at both the excitation wavelength $(650 \mathrm{~nm})$ and the emission wavelength $(700 \mathrm{~nm})$. We assigned the fluorophore concentration to be 1 in the target regions and 0 in the background regions. Like the numerical simulation studies described above, we added 30\% Gaussian noise to the numerical FMT measurement data.

In the kernel method, we extracted the feature vectors from the MRI images easily because the finite element mesh was generated from the same MRI images. Then, we generated the kernel matrix using the Eqs. (8) and (9) to incorporate anatomical information from MRI images into the FMT reconstruction by minimizing the kernelized objective function as described in Eq. (13).

The voxel number for each corresponding node and the number of nearest neighbors in knnsearch are important parameters in generating the kernel matrix $\mathbf{K}$ and have significant effects on the kernel reconstruction method. In this paper, we studied three different voxel numbers, $3 \times 3 \times 3,5 \times 5 \times 5$, and $7 \times 7 \times 7$. The lengths of feature vectors were 27,125 , and 343 , respectively. For knnsearch, different values of $k(16,32,64,128$, 256), the number of nearest neighbors, were also studied.

During the FMT reconstruction, for the kernel method, we used the MRI images without segmentation as the anatomical guidance to generate the $\mathbf{K}$ matrix. For the soft prior method, we used the segmented images to generate the soft prior matrix without adding any segmentation error.

\subsection{Phantom Experimental Setup}

To validate our algorithm, we conducted an agar phantom experiment. In this experiment, we used a cylindrical phantom with a diameter of $22 \mathrm{~mm}$ and a length of $80 \mathrm{~mm}$. The phantom was composed of $1 \%$ intralipid, $2 \%$ agar, $20 \mu \mathrm{M}$ bovine hemoglobin (H2625, Sigma-Aldrich Inc., St. Louis, Missouri), and water. We embedded two capillary tubes with a length of $20 \mathrm{~mm}$ and a diameter of $1.4 \mathrm{~mm}$ as targets, in which $20 \mu \mathrm{M}$ SulfoCyanine5 dye (Lumiprobe Corporation, Hallandale Beach, Florida) was injected. The geometry of the experimental phantom with two targets is shown in Fig. 2, where the two red bars indicate two FMT targets. The edge-to-edge distance of the two targets was $2.94 \mathrm{~mm}$.

The phantom geometry was discretized with a 3-D tetrahedral finite element mesh with 37,333 nodes and 199,881 elements. During the FMT imaging, a line laser (1-mm wide and 50-mm long) at the wavelength of $643 \mathrm{~nm}$ scanned the surface of the phantom sequentially with 30 excitation positions which were distributed uniformly on the phantom surface. For each line laser excitation position, an emission picture at the wavelength of $720 \mathrm{~nm}$ was taken. All 9384 surface nodes on the side surface of the cylinder were used as the detector nodes, and the measurements were obtained from the acquired 


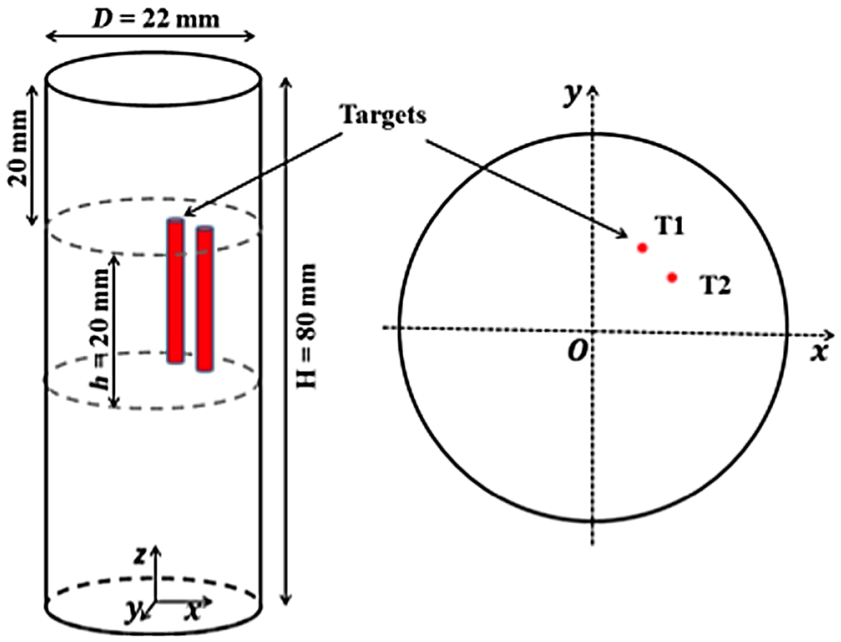

Fig. 2 The geometry of the phantom experiment with target locations at T1 (1.72 and 4.71) and T2 (5.01 and 1.87).

emission pictures. Details of the conical mirror-based FMT imaging system were described in Ref. 52. The phantom optical properties were $\mu_{a}=0.012 \mathrm{~mm}^{-1}, \mu_{s}^{\prime}=0.83 \mathrm{~mm}^{-1}$ at both 643 and $720 \mathrm{~nm}$ wavelengths.

We scanned the phantom with our lab-made micro-CT imaging system with 180 projections ${ }^{53}$ and reconstructed the 3-D CT images of the phantom with an isotropic voxel size of $0.15 \mathrm{~mm}$. The micro-CT system consisted of an x-ray source and a flat panel detector placed opposite to each other on a micro-CT gantry that rotated around the bed, where the phantom was placed. The source-to-isocenter distance was $205.34 \mathrm{~mm}$, and the source-to-detector distance was $246.2 \mathrm{~mm}$. The detector had a $49.2 \mathrm{~mm}$ by $49.2 \mathrm{~mm}$ sensing area consisting of 1024 by 1024 pixel sensors with $48 \mu \mathrm{m}$ pixel spacing. The $\mathrm{x}$-ray tube was operated at a current of $0.5 \mathrm{~mA}$ and a voltage of $50 \mathrm{kVp}$. A filtered-backprojection algorithm was used to reconstruct the micro-CT images with a Shepp-Logan filter. The obtained CT images are shown in Fig. 9(a), from which we calculated the targets' size and position. Because Sulfo-Cyanine5 dye does not have CT contrast, only the capillary tubes were observed in the reconstructed micro-CT images, and the fluorescence dye (target) region were filled by pixels having the same CT contrast as the capillary tube. Then, the modified micro-CT images were used as anatomical guidance in the kernel method.

\subsection{FMT Image Evaluation Criteria}

According to our previous studies, the combinations of four metrics listed below can evaluate the quality of the reconstructed FMT images very well. Their detailed definitions can be found in Refs. 39 and 40. Briefly, the volume ratio (VR) measures the ratio between the true region of interest and the reconstructed region of interest. The Dice similarity coefficient (Dice) measures the location accuracy of the reconstructed target. Ideally, VR and Dice coefficients should be 1 . The contrastto-noise ratio (CNR) measures how well the reconstructed target is distinguished from its background. The higher the CNR coefficient, the better the reconstructed image. The mean square error (MSE) is the difference between the measurements and the model predictions. The MSE closer to zero is better.

\section{Results}

\subsection{Simulation Results}

\subsubsection{Numerical simulation with two FMT targets}

In this simulation, we had two capillary tube targets embedded inside the cylindrical background phantom with an edge-to-edge distance of $2 \mathrm{~mm}$ as described in Fig. 1(a). For comparison, we have reconstructed FMT images with the soft prior method. The ground-truth FMT images, simulated CT images, and the reconstructed FMT images with the soft prior method are shown in Fig. 3. All the FMT reconstructions in this paper were conducted in 3-D, and the reconstructed FMT images are shown by slices along the $z$-axis with equal interval. Then, we performed the reconstruction with the proposed kernel-based FMT reconstruction algorithm. To investigate how the parameters in the kernel method affect the FMT reconstruction, we studied three different voxel numbers $(3 \times 3 \times 3,5 \times 5 \times 5$, and $7 \times 7 \times 7)$ and three different nearest neighbors $(k=16,32$, 64 ) with nine combinations of the kernel-based FMT reconstructions. The reconstructed FMT images are shown in Fig. 4, in which each column indicates different voxel numbers and each row indicates different numbers of nearest neighbors. For all nine cases, the two targets have been reconstructed and separated successfully as indicated in Fig. 4.

To evaluate the simulation results quantitatively, we calculated image quality metrics, such as VR, Dice, CNR, and MSE for the FMT reconstruction with the soft prior method,

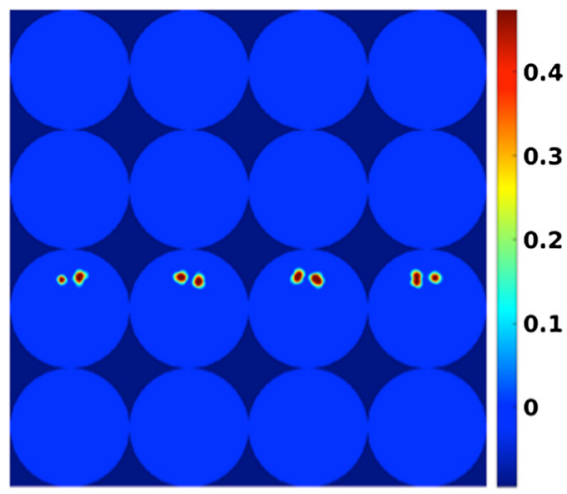

(a)

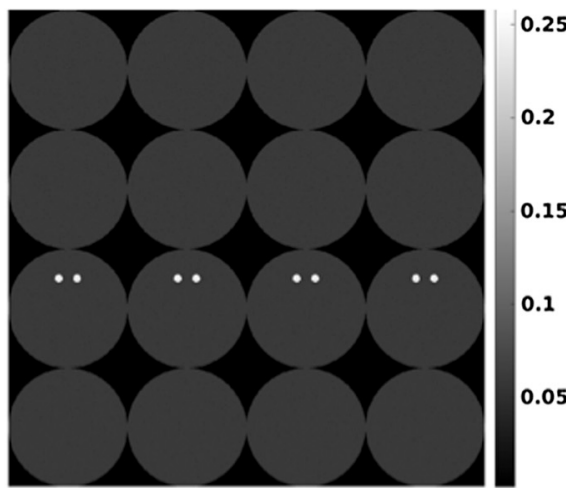

(b)

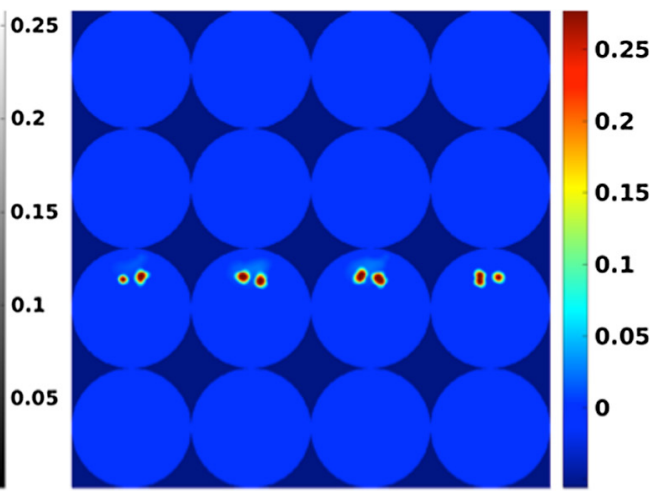

(c)

Fig. 3 For the numerical simulation of two targets: (a) the ground-truth images, (b) simulated anatomical guidance images, and (c) the reconstructed FMT images with the soft prior method. The interval between slices along $z$-axis is $5.33 \mathrm{~mm}$. 


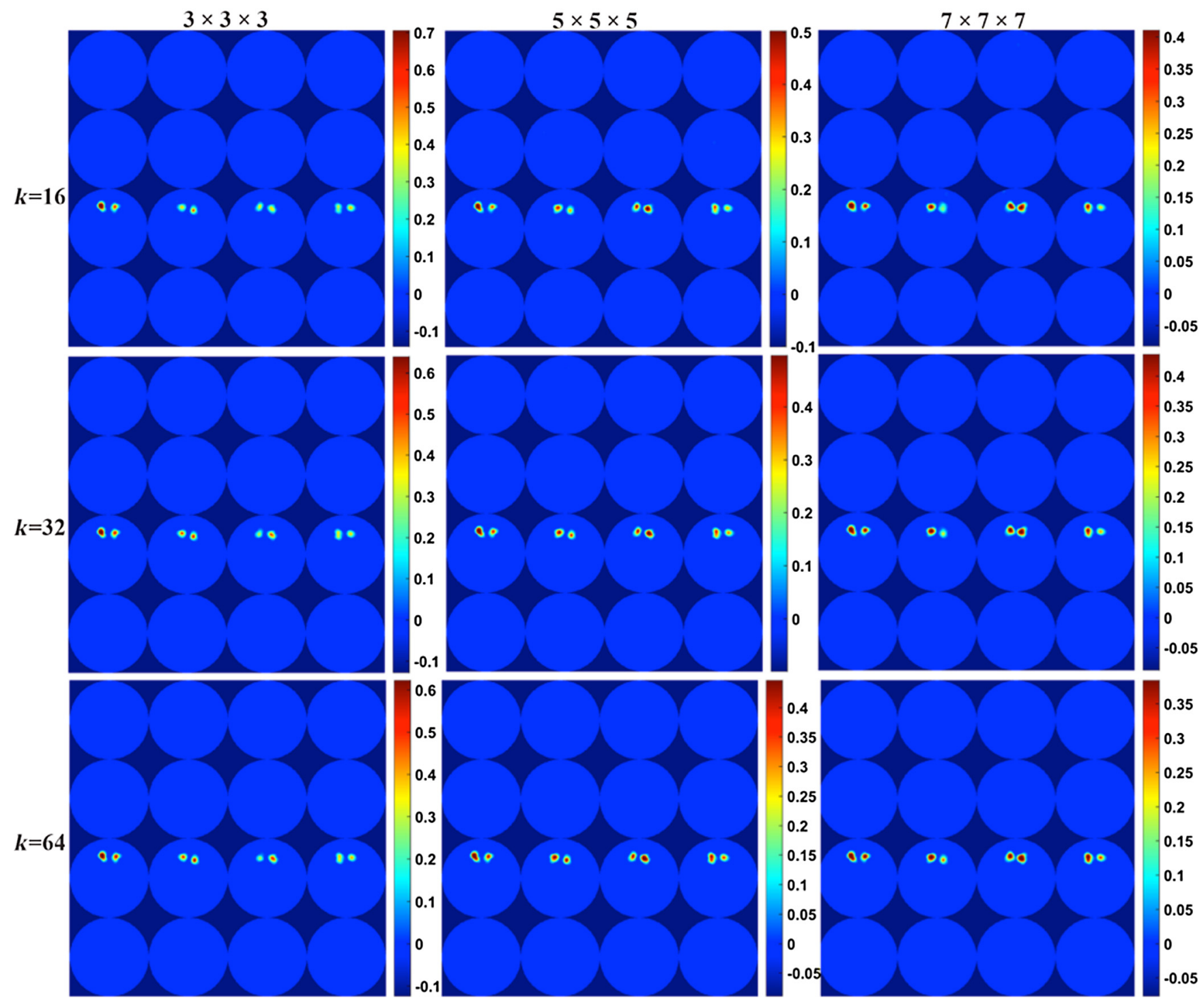

Fig. 4 Reconstruction FMT images for the cylindrical phantom simulation of two targets by the kernel method with different nearest neighbor $k$ as indicated by each row and different voxel numbers indicated by each column. The interval between slices along $z$-axis is $5.33 \mathrm{~mm}$.

and the nine FMT reconstructions with the kernel method, as shown in Table 1. For the kernel method, when the voxel number is fixed, we have better FMT reconstruction quality as the nearest neighbor $k$ increases. One example is that the Dice increased from 0.02 to 0.23 as $k$ increased from 16 to 64 for the voxel number of $3 \times 3 \times 3$. Similarly, for the fixed nearest neighbor $k$, we found that the FMT image quality becomes better with a larger voxel number. The best FMT reconstruction result was obtained with $k=64$ and a voxel number of $7 \times 7 \times 7$, which is highlighted in Table 1 . From Table 1 , we see that the soft prior method performed better than the kernel method in this simulation when the target regions were known accurately in the anatomical guidance.

\subsubsection{Elliptic cylindrical phantom simulation with two FMT targets}

In this simulation, we had two capillary tube targets embedded inside the elliptic cylindrical background phantom with an edge-to-edge distance of $1 \mathrm{~mm}$ as described in Fig. 1(b). For comparison, we have also reconstructed FMT images with the soft prior method. The ground-truth FMT images, the simulated CT images, and the reconstructed FMT images with the soft prior method are shown in Fig. 5. Then, we performed the reconstruction with the proposed kernel-based FMT reconstruction algorithm. To investigate how the parameters in the kernel method affect the FMT reconstruction, we studied three different voxel numbers $(3 \times 3 \times 3,5 \times 5 \times 5$, and $7 \times 7 \times 7)$ and three different nearest neighbors $(k=64,128$, $256)$ with nine combinations of the kernel-based FMT reconstructions. The reconstructed FMT images with the kernel method are shown in Fig. 6, in which each column indicates different voxel numbers and each row indicates different numbers of nearest neighbors. For all nine cases, the two targets have been reconstructed and separated successfully as indicated in Fig. 6.

To evaluate the simulation results quantitatively, we calculated quantitative image quality metrics for the FMT 
Table 1 For the cylindrical phantom simulation of two targets, the calculated VR, Dice, CNR, and MSE with the kernel method for different numbers of nearest neighbor $k$ and different voxel numbers, and with the soft prior method.

\begin{tabular}{cccccc}
$k$ & Voxel number & VR & Dice & CNR & MSE \\
\hline 16 & $3 \times 3 \times 3$ & 0.124 & 0.002 & 18.264 & $3.88 \times 10^{-4}$ \\
16 & $5 \times 5 \times 5$ & 0.223 & 0.159 & 21.351 & $2.96 \times 10^{-4}$ \\
16 & $7 \times 7 \times 7$ & 0.387 & 0.306 & 19.554 & $3.44 \times 10^{-4}$ \\
32 & $3 \times 3 \times 3$ & 0.127 & 0.006 & 19.688 & $3.41 \times 10^{-4}$ \\
32 & $5 \times 5 \times 5$ & 0.243 & 0.187 & 22.713 & $2.51 \times 10^{-4}$ \\
32 & $7 \times 7 \times 7$ & 0.451 & 0.430 & 22.099 & $2.66 \times 10^{-4}$ \\
64 & $3 \times 3 \times 3$ & 0.139 & 0.23 & 20.029 & $3.29 \times 10^{-4}$ \\
64 & $5 \times 5 \times 5$ & 0.347 & 0.323 & 23.956 & $2.22 \times 10^{-4}$ \\
64 & $7 \times 7 \times 7$ & 0.639 & 0.596 & 24.111 & $2.21 \times 10^{-4}$ \\
& Soft prior & 0.952 & 0.964 & 32.355 & $1.67 \times 10^{-4}$ \\
\hline
\end{tabular}

Note: Bold values indicate the best case of the kernel method.

reconstruction with the soft prior method and the nine FMT reconstructions with the kernel method, as shown in Table 2. From Table 2, when the voxel number is fixed, we have better FMT reconstruction quality as the nearest neighbor $k$ increases. One example is that the Dice increased from 0.469 to 0.803 as $k$ increased from 64 to 256 for the voxel number of $3 \times 3 \times 3$. Similarly, for the fixed nearest neighbor $k$, we found that the FMT image quality becomes better with larger voxel number in this simulation setup. The best FMT reconstruction result was obtained with $k=256$ with $7 \times 7 \times 7$ voxel size, which is highlighted in Table 2.

\subsubsection{Numerical simulation with false target size in the numerical anatomical CT images}

The numerical phantom geometry of this simulation study, same as the second simulation, is shown in Fig. 1(b). However, the diameter of the right target in the simulated anatomical guidance CT images [Fig. 7(b)] was enlarged intentionally from 1.4 to $2.8 \mathrm{~mm}$ to study how the false target size affects the FMT reconstruction with the proposed kernel method. The enlarged target was moved to the right side for $0.7 \mathrm{~mm}$, so that the edgeto-edge distance of the two targets was still $1 \mathrm{~mm}$ in the simulated CT images as shown in Fig. 7(b). For comparison, we performed the FMT reconstruction with the soft prior method and with the kernel method of three different nearest neighbor $k$ and three different voxel numbers as in the above section. Among all the reconstructions with the kernel method, unlike the previous simulation, we found that the reconstruction with the nearest neighbor of $k=256$ and the voxel number of $3 \times 3 \times 3$ had the least error with an MSE of $8.54 \times 10^{-4}$, whereas the soft prior method had the MSE of $1.31 \times 10^{-3}$. Figure 7 plots the ground-truth images [Fig. 7(a)], the reconstructed FMT images with soft prior method [Fig. 7(c)], and the reconstructed FMT images by the kernel method with the nearest neighbor of $k=$ 256 and the voxel number of $3 \times 3 \times 3$ [Fig. 7(d)]. As indicated in Fig. 7(c), the two targets were barely separated with the soft prior method. Figure 7(d) is a representative reconstructed FMT image with the kernel method and indicates that the image quality is much better than that of Fig. 7(c) as demonstrated by the CNR of 12.214 for Fig. 7(c) and 17.543 for Fig. 7(d). The calculated image quality metrics are listed in Table 3.

\subsubsection{Numerical simulation using MRI images of a rat brain}

To validate the proposed kernel-based FMT image reconstruction algorithm with guidance from realistic anatomical images, we conducted this simulation study using in vivo rat brain MRI images as shown in Fig. 8(a). Details of the MRI images and the simulation setup are described in Sec. 2.4.3. The contrast of the brain tumor to its surrounding tissues in the MRI images is 2:1 approximately. First, we reconstructed the FMT images using the soft prior method, in which only two regions were considered. We have obtained very good FMT results from the soft prior method as shown in Fig. 8(b). Figure 8(c) plots the reconstructed FMT images obtained by the kernel method using the MRI images as the anatomical guidance directly without segmentation. In the kernel method, we set the nearest neighbor of $k=256$ and the voxel number of $3 \times 3 \times 3$. From Fig. 8 ,

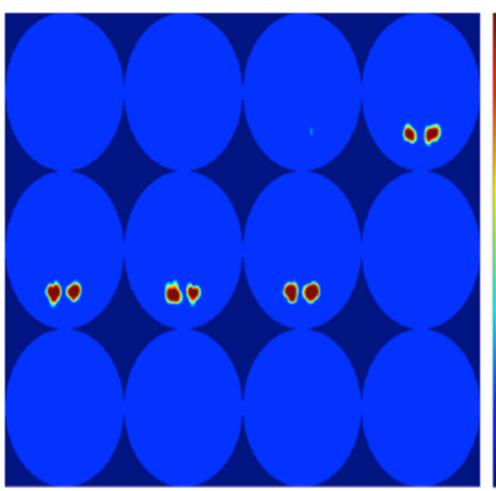

(a)

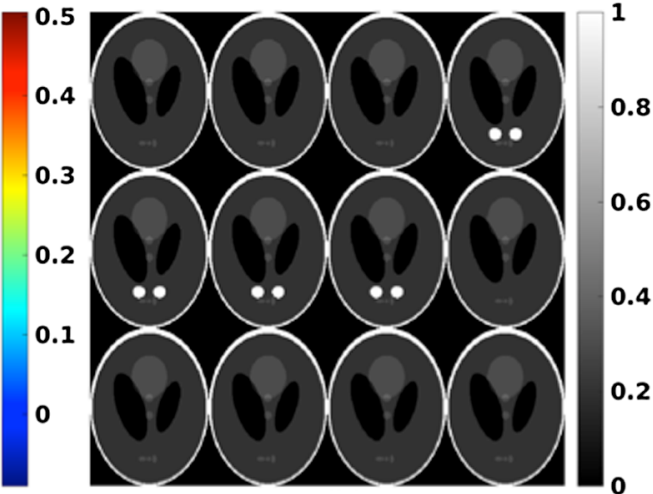

(b)

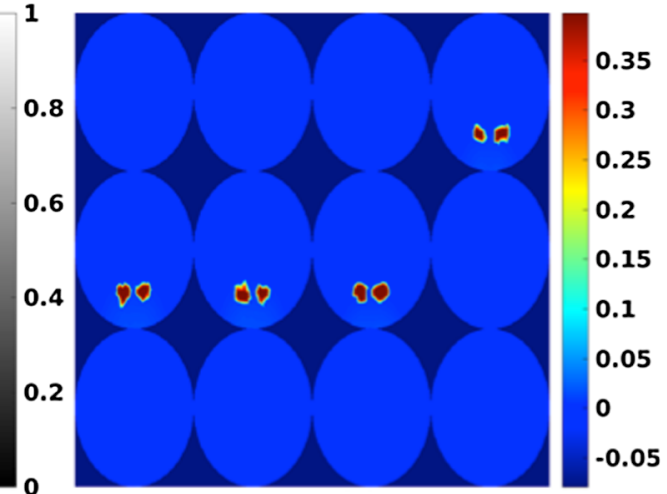

(c)

Fig. 5 For the simulation of elliptic cylindrical phantom with two FMT targets, (a) the ground-truth images, (b) simulated CT images, and (c) the reconstructed FMT images with the soft prior method. The interval between slices along $z$-axis is $4.54 \mathrm{~mm}$. 

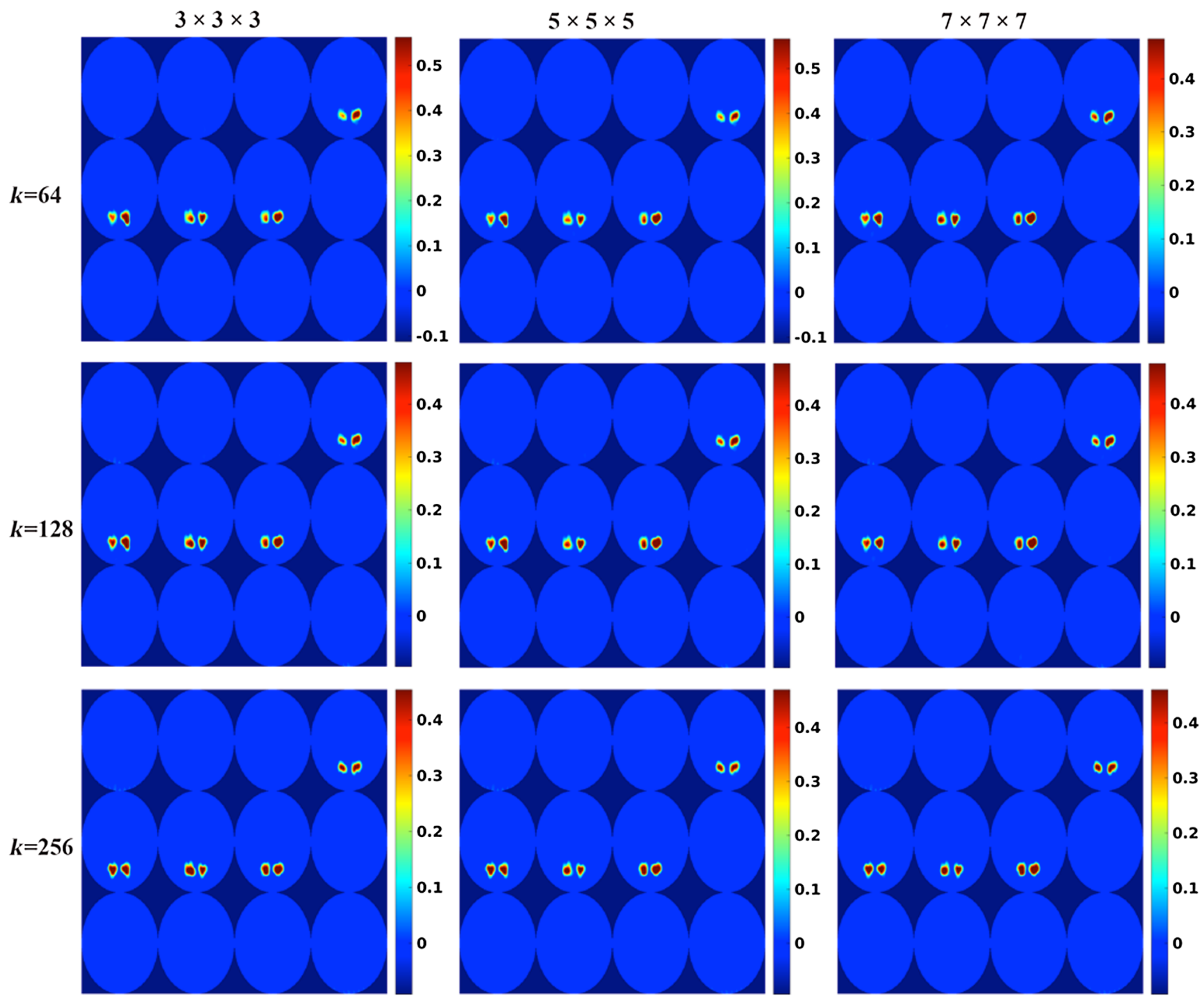

Fig. 6 Reconstructed FMT images for the elliptic cylindrical phantom simulation of two targets by the kernel method with different nearest neighbor $k$ as indicated by each row and different voxel numbers indicated by each column. The interval between slices along $z$-axis is $4.54 \mathrm{~mm}$.

we can see that the kernel method has reconstructed the target very well with comparable results from the soft prior method. The VR, Dice, CNR, and MSE are 0.529, 0.626, 23.007, and $7.14 \times 10^{-4}$ for the kernel method, and $0.966,0.974,42.622$, and $3.12 \times 10^{-4}$ for the soft prior method, respectively.

\subsection{Phantom Experimental Results}

\subsubsection{Reconstruction with homogeneous background in CT images}

The phantom's geometry is shown in Fig. 2. As described in the numerical simulation section, we have performed the FMT reconstruction of this phantom experiment with the soft prior method and with kernel method of 15 different cases with five different nearest neighbor $k(16,32,64,128,256)$ and three different voxel numbers $(3 \times 3 \times 3,5 \times 5 \times 5,7 \times 7 \times 7)$. The reconstructed FMT images along with anatomical CT images are shown in Fig. 9. The kernel-based reconstruction results [Fig. 9(d)] are as good as the results from the soft prior method [Fig. 9(c)] when the homogeneous anatomical images were used as the guidance. For comparison, we have also reconstructed the target without anatomical guidance as shown in Fig. 9(b), from which we see that the two targets were reconstructed with large position errors. To analyze the reconstructed FMT images quantitatively, we have calculated the VR, Dice, and CNR as listed in Table 4 for each case, where the micro-CT images were referred as the ground-truth images when we calculated the image quality metrics. The MSE has not been calculated because we do not know the exact fluorescent dye concentration. From Table 4, we know that the kernel method can achieve good reconstruction results with the nearest neighbor of 64 and the voxel number of $5 \times 5 \times 5$, in which the VR, Dice, and CNR are 0.714, 0.643, and 25.849, respectively. The VR, Dice, and CNR are 0.729, 0.757, and 30.312 for the FMT reconstruction with the soft prior method. These similar image quality metrics indicate that the kernel method is as good as the soft prior method for the FMT reconstruction with the homogeneous background. 
Table 2 For the numerical simulation of elliptic cylindrical phantom with two FMT targets, the calculated VR, Dice, CNR, and MSE with the kernel method for different numbers of nearest neighbor $k$ and different voxel numbers, and with the soft prior method.

\begin{tabular}{lccccc}
$k$ & Voxel number & VR & Dice & CNR & MSE \\
\hline 64 & $3 \times 3 \times 3$ & 0.366 & 0.469 & 18.971 & $6.22 \times 10^{-4}$ \\
64 & $5 \times 5 \times 5$ & 0.318 & 0.419 & 19.452 & $6.16 \times 10^{-4}$ \\
64 & $7 \times 7 \times 7$ & 0.519 & 0.635 & 22.262 & $4.82 \times 10^{-4}$ \\
128 & $3 \times 3 \times 3$ & 0.516 & 0.604 & 20.811 & $5.16 \times 10^{-4}$ \\
128 & $5 \times 5 \times 5$ & 0.481 & 0.590 & 21.113 & $5.39 \times 10^{-4}$ \\
128 & $7 \times 7 \times 7$ & 0.519 & 0.653 & 23.072 & $4.57 \times 10^{-4}$ \\
256 & $3 \times 3 \times 3$ & 0.713 & 0.803 & 25.028 & $1.35 \times 10^{-4}$ \\
256 & $5 \times 5 \times 5$ & 0.668 & 0.782 & 25.286 & $1.46 \times 10^{-4}$ \\
256 & $7 \times 7 \times 7$ & 0.757 & 0.845 & 26.108 & $1.21 \times 10^{-4}$ \\
& Soft prior & 1.046 & 0.934 & 26.661 & $1.73 \times 10^{-4}$ \\
\hline
\end{tabular}

Note: Bold values indicate the best case of the kernel method.
Table 3 For the numerical simulation with a false target size, the calculated VR, Dice, CNR, and MSE with the kernel method of nearest neighbor $k=256$ and different voxel numbers, and with the soft prior method.

\begin{tabular}{lccccc}
$k$ & Voxel number & VR & Dice & CNR & MSE \\
\hline 256 & $3 \times 3 \times 3$ & $\mathbf{0 . 3 9 4}$ & $\mathbf{0 . 5 4 8}$ & $\mathbf{1 7 . 5 4 3}$ & $\mathbf{8 . 5 4 \times 1 0 ^ { - 4 }}$ \\
256 & $5 \times 5 \times 5$ & 0.281 & 0.416 & 16.816 & $8.91 \times 10^{-4}$ \\
256 & $7 \times 7 \times 7$ & 0.409 & 0.537 & 16.728 & $9.06 \times 10^{-4}$ \\
& Soft prior & 0.466 & 0.527 & 12.214 & $1.31 \times 10^{-3}$ \\
\hline
\end{tabular}

Note: Bold values indicate the best case of the kernel method.

\subsubsection{Reconstruction with inhomogeneous background in CT images}

To further validate the proposed method with guidance from complex anatomical images, we added some artificial features in the physical CT images we obtained. As shown in Fig. 10(a), the darkest big cylinder has an intensity of $<50 \%$ of the background. The other two big cylinders have an intensity of $50 \%$ more than the background intensity. We also added another three small cylinders at the random locations with different intensities.

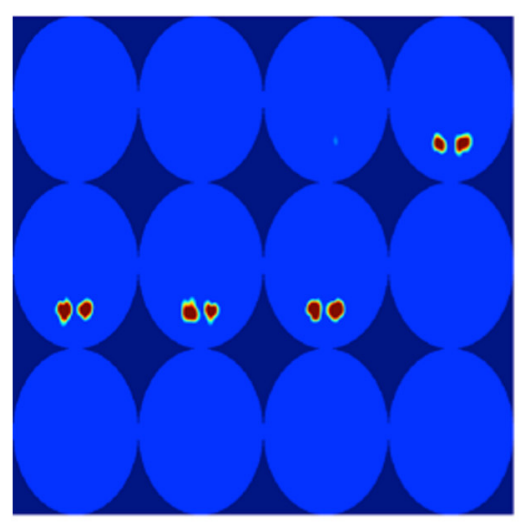

(a)

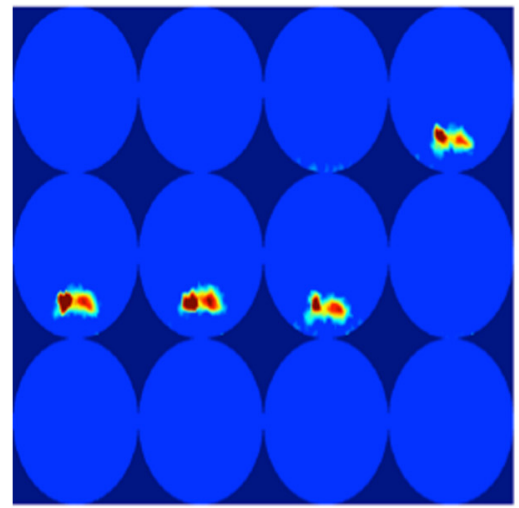

(c)
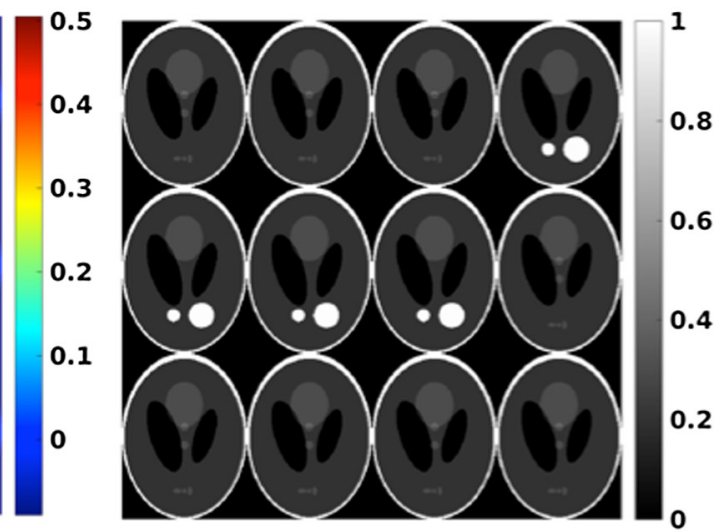

(b)

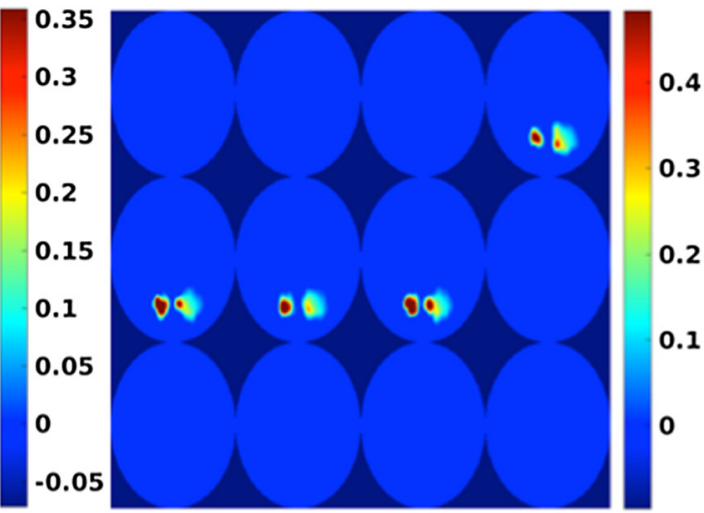

(d)

Fig. 7 For the numerical simulation with false larger target size, (a) the ground-truth images, (b) simulated CT images with the false enlarged target, (c) the reconstructed FMT images with the soft prior method, and (d) the reconstructed FMT images by the kernel method with the nearest neighbor of $k=256$ and the voxel number of $3 \times 3 \times 3$. The interval between slices along $z$-axis is $4.54 \mathrm{~mm}$. 


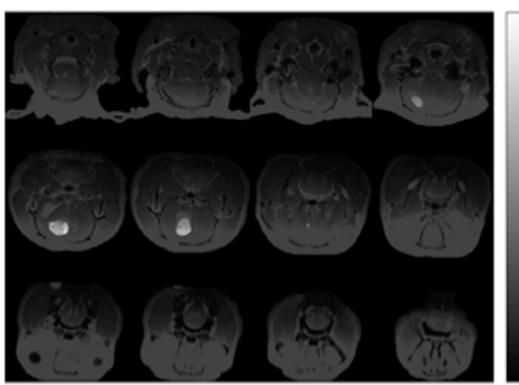

(a)

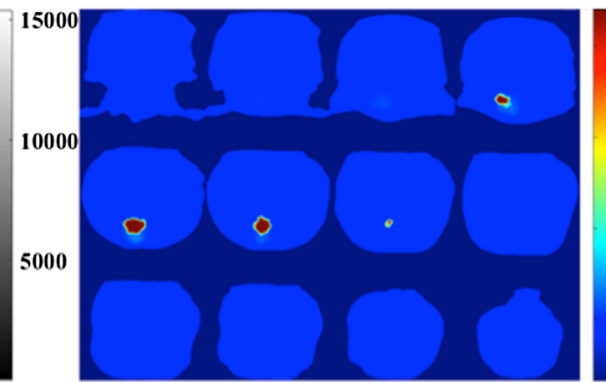

(b)

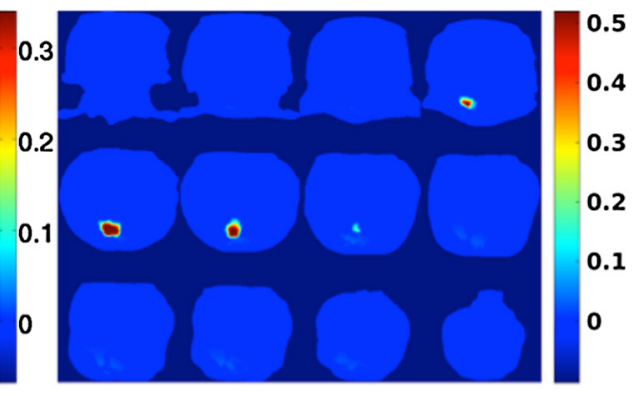

(c)

Fig. 8 Numerical simulation with the rat brain MRI images: (a) MRI images, (b) the reconstructed FMT images with the soft prior method, and (c) the reconstructed FMT images with the kernel method with $k=256$ and the voxel number of $3 \times 3 \times 3$. The interval between slices along $z$-axis is $2.45 \mathrm{~mm}$.

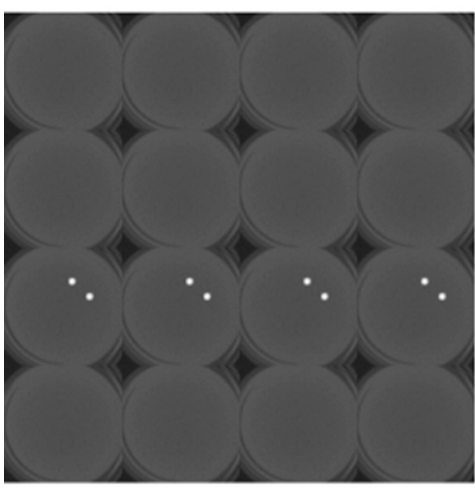

(a)

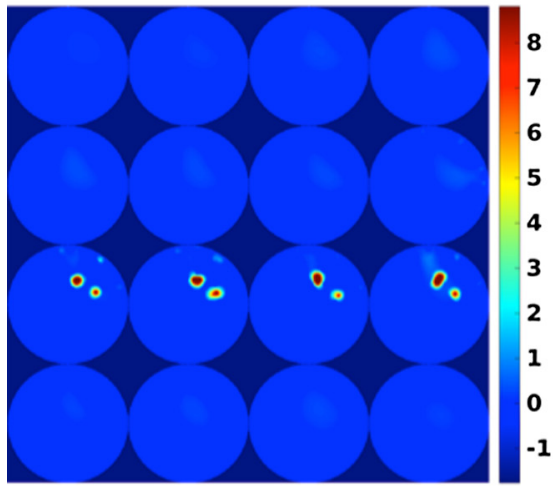

(c)

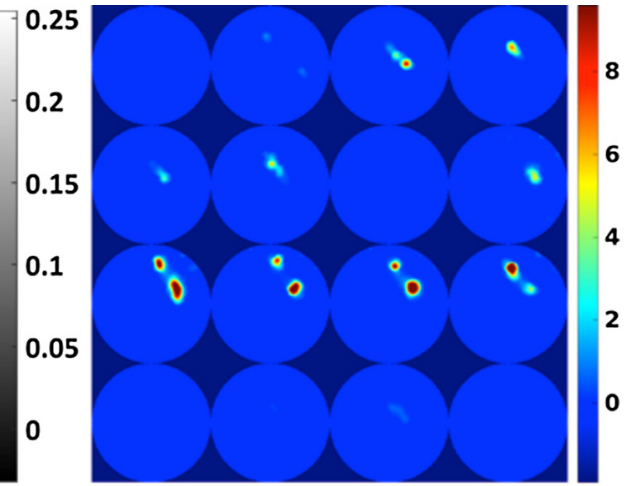

(b)

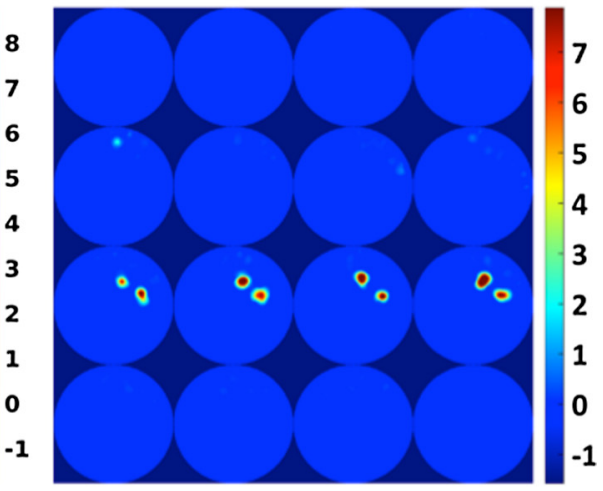

(d)

Fig. 9 (a) Original CT images. The reconstructed FMT images (b) without priors, (c) with the soft prior method and the homogenous background, (d) with the kernel method using original CT images as guidance with $k=64$ and the voxel number of $5 \times 5 \times 5$. The interval between slices along $z$-axis is $5.33 \mathrm{~mm}$.

Table 4 The calculated VR, Dice, and CNR for the phantom experiments without prior, with the kernel method, and with the soft prior method.

\begin{tabular}{lcccccccc}
\hline & \multicolumn{3}{c}{ Homogeneous } & & & \multicolumn{3}{c}{ Inhomogeneous } \\
\cline { 2 - 3 } & VR & Dice & CNR & & VR & Dice & CNR \\
\hline Soft prior & 0.729 & 0.757 & 30.312 & & 0.677 & 0.728 & 29.704 \\
Kernel method & 0.714 & 0.643 & 25.849 & & 0.672 & 0.648 & 28.250 \\
No prior & 0.752 & 0.025 & 2.569 & & & \\
\hline
\end{tabular}

Two of them have an intensity of five times more than the background, which is slightly higher than the targets' intensity. Those bright inclusions mimic bones in the CT images or fat and blood in the MRI images. For the FMT reconstruction with the soft prior method, we had eight regions: two targets, two bright artificial feature, three big cylinder artificial features, and the background. Unlike the homogeneous background case, here we obtained the best kernel-method-based FMT images with the nearest neighbor of 256 and the voxel number of $3 \times 3 \times 3$, in which the VR, Dice, and CNR are 0.672, 0.648, and 28.250 , respectively. These image quality metrics are slightly lower than those of the soft prior method, which are 0.677 , 


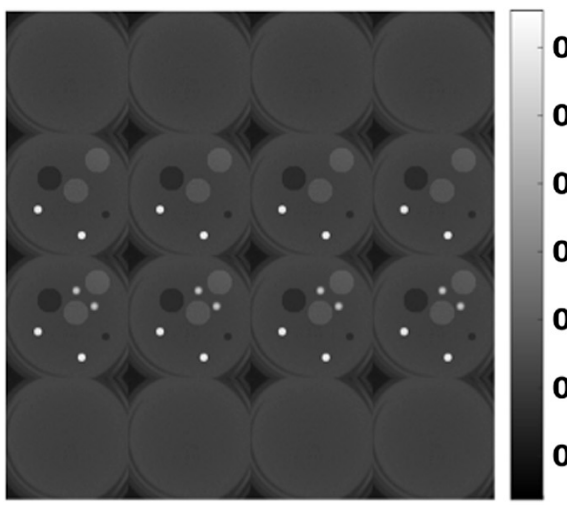

(a)

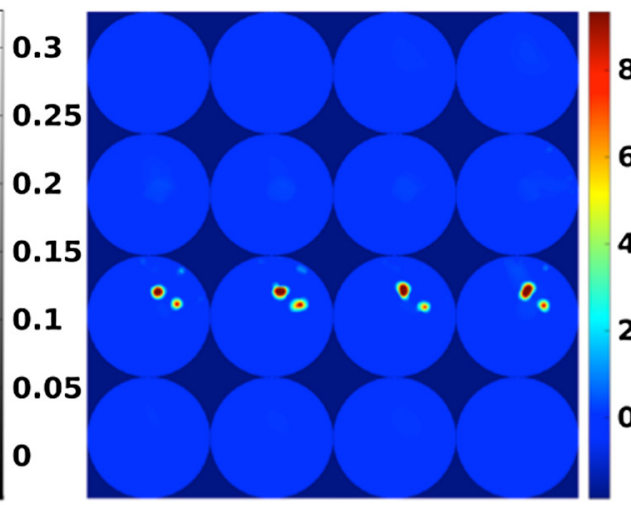

(b)

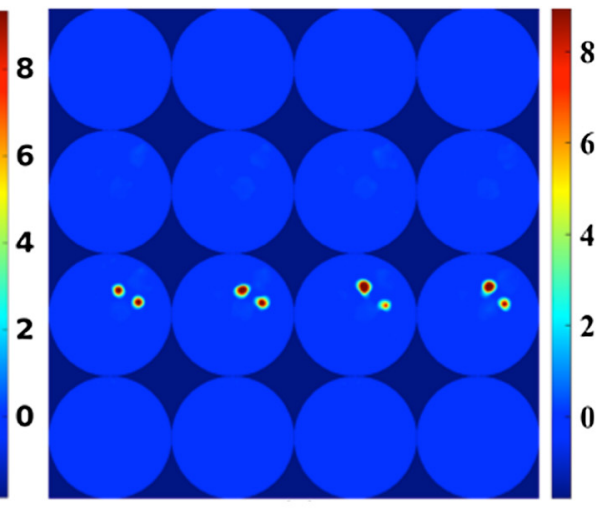

(c)

Fig. 10 (a) CT images with artificial features. (b) Reconstructed FMT images with the soft prior method and the inhomogenous background in the CT images. (c) Reconstructed FMT images with the kernel method using the CT images with artificial features as the guidance with $k=256$ and the voxel number of $3 \times 3 \times 3$. The interval between slices along $z$-axis is $5.33 \mathrm{~mm}$.

0.728 , and 29.704, respectively. These results demonstrated that the kernel method is able to achieve comparable results as the soft prior method when there are inhomogeneous inclusions in the anatomical guidance images.

\section{Discussions and Conclusion}

In this paper, we have introduced the kernel method as an approach to including the anatomical guidance in the FMT image reconstruction, in which a kernel matrix having the anatomical priors is created and incorporated into the projection model of FMT. It is worth noting that we used the forward model without the kernel method to generate the FMT measurements for numerical simulations. Numerical simulations and phantom experiments have been performed to demonstrate that the proposed kernel method has reconstructed the FMT targets successfully and have comparable results as the soft prior method.

Compared with conventional Laplacian-type regularization method to include anatomical priors such as the soft prior, the kernel method has the advantage of easy implementation, in which we do not need to segment the target and background regions in the anatomical images. This advantage is more significant for some cases in which the targets are not easily differentiated and segmented. This may result in a concern of the misguidance from the false-positive regions. To address this issue, we have performed one numerical simulation with a false target size (from 1.4 to $2.8 \mathrm{~mm}$ in diameters) as described in Sec. 3.1.3. Our results indicate the false target size guidance has some effects when two targets are very close. However, the kernel method performs better than the soft prior method. Another advantage of the kernel method is that, because we set the regularization parameter to be zero, we do not have to search for the optimum regularization parameter, which is searched with the $L$-curve method in conventional regularization methods.

To generate the kernel matrix, three parameters must be set before the FMT image reconstruction. The first parameter is the Gaussian kernel coefficient $\sigma$. According to previous studies, ${ }^{38,49} \sigma=1$ yields best results. The second parameter is the number of nearest neighbor's $k$. From the results of both numerical simulations and phantom experiments, we found that the reconstructed image quality is better with a larger number of $k$. It also depends on the features of anatomical images. As indicated in the numerical simulations with cylindrical geometry and the phantom experiment with homogeneous background, for anatomical images with fewer features, we can obtain good reconstruction results considering only 64 nearest neighbors in the kernel matrix. However, for the anatomical images with rich features, such as the elliptic cylindrical simulation, the simulation with MRI data and the phantom experiment with inhomogeneous background, we have to consider more of the nearest neighbors in the kernel matrix. We also found that for the simulations and experiments with the kernel method of $k=64$, we can obtain better reconstruction results with $k=$ 256 at a price of longer time to form the kernel matrix. The third parameter is the voxel number. As demonstrated in the first and second simulations, for the anatomical images without any false information, the quality of reconstructed FMT images increases slightly as the voxel number increases for a fixed number of nearest neighbors $k$. However, for the numerical simulations with the false target size in anatomical images, the kernel method with the smaller number of voxels performed better than the kernel method with the larger number of voxels. As shown in Table 3, Dice and CNR are achieved highest with the voxel number of $3 \times 3 \times 3$ for the reconstruction with the kernel method when $k=256$. MSE also reached the lowest for the case of voxel number $3 \times 3 \times 3$. VR coefficient is not informative in this case because the incorrect bigger size of the target in the anatomical images introduces higher VR to the reconstruction images. Similarly, the numerical simulations with the MRI images and the phantom experiment with artificial features have further demonstrated this trend by obtaining the best results with of voxel number of the $3 \times 3 \times 3$.

The kernel matrix was generated before the FMT reconstruction and the matrix generation time depends on the voxel size

Table 5 Time to generate the kernel matrix $\mathbf{K}$ with different $k$ and voxel sizes (in seconds).

\begin{tabular}{lrrrrr} 
Voxel size & $k=16$ & $k=32$ & $k=64$ & $k=128$ & $k=256$ \\
\hline $3 \times 3 \times 3$ & 3.36 & 3.78 & 5.46 & 8.88 & 20.09 \\
$5 \times 5 \times 5$ & 11.32 & 12.78 & 16.81 & 23.58 & 38.79 \\
$7 \times 7 \times 7$ & 28.89 & 34.59 & 43.05 & 57.30 & 96.42 \\
\hline
\end{tabular}


and the nearest neighbor number. Table 5 lists the $\mathbf{K}$ matrix generation time for the elliptic cylindrical simulation with two targets. For the best image quality setup with the nearest neighbor of 256 and the voxel number of $3 \times 3 \times 3$, the $\mathbf{K}$ matrix generation time was $20.09 \mathrm{~s}$ on a cluster with 12 nodes $(2.8 \mathrm{GHz}$ each node) and 128 GB memory. This is slightly longer than the time of generating the regularization matrix for the soft prior method, which was $12.28 \mathrm{~s}$ in this simulation with five regions. In this study, the kernel-method-based FMT reconstruction converged in no more than 10 iterations, which were around $5 \mathrm{~s}$ in total.

The grayscale values in the anatomical guidance images are included in the kernel matrix so that these values affect the kernel method. As shown in the rat brain case, when the target has distinct contrast to other background regions, the kernel method performed very well. And we have also found that the kernel method is robust to the inhomogeneous inclusions in the anatomical guidance images when these inclusions have lower grayscale values than the values in the target region. However, it is fine to have inclusions with larger grayscales values when the inclusions are not close to the target as shown in Fig. 10(a).

In summary, we have introduced a kernel-method-based FMT reconstruction algorithm as a new approach to include the anatomical guidance. Numerical simulations prove that this method is robust in overcoming incorrect anatomical guidance. Phantom experiments further validate that the proposed method can improve the FMT reconstruction quality and does not increase the reconstruction time. In the future, we will apply the proposed kernel method to in vivo experiments on the hybrid systems.

\section{Disclosures}

No conflicts of interest, financial or otherwise, are declared by the authors.

\section{Acknowledgments}

Authors thank research support from the startup fund, UC Merced (C. Li) and National Institute of Health; award no. CA 134659 (PI: Katherine W. Ferrara). Authors thank Dr. Guobao Wang from Department of Radiology, UC Davis, and Dr. Jinyi Qi from Department of Biomedical Engineering, UC Davis, for helpful discussions.

\section{References}

1. S. R. Cherry, "In vivo molecular and genomic imaging: new challenges for imaging physics," Phys. Med. Biol. 49(3), R13-R48 (2004).

2. V. Ntziachristos et al., "Looking and listening to light: the evolution of whole-body photonic imaging," Nat. Biotechnol. 23(3), 313-320 (2005).

3. S. C. Davis et al., "MRI-coupled fluorescence tomography quantifies EGFR activity in brain tumors," Acad. Radiol. 17(3), 271-276 (2010).

4. X. Guo et al., "A combined fluorescence and microcomputed tomography system for small animal imaging," IEEE Trans. Biomed. Eng. 57(12), 2876-2883 (2010).

5. S. C. Davis et al., "Dynamic dual-tracer MRI-guided fluorescence tomography to quantify receptor density in vivo," Proc. Natl. Acad. Sci. U. S. A. 110(22), 9025-9030 (2013).

6. Y. Zhang et al., "In vivo tomographic imaging with fluorescence and MRI using tumor-targeted dual-labeled nanoparticles," Int. J. Nanomed. 9(1), 33-41 (2014).

7. A. Ale et al., "FMT-XCT: in vivo animal studies with hybrid fluorescence molecular tomography-X-ray computed tomography," Nat. Methods 9(6), 615-620 (2012).
8. X. Ma et al., "Assessment of asthmatic inflammation using hybrid fluorescence molecular tomography-x-ray computed tomography," J. Biomed. Opt. 21(1), 015009 (2016).

9. A. Corlu et al., "Three-dimensional in vivo fluorescence diffuse optical tomography of breast cancer in humans," Opt. Express 15(11), 66966716 (2007).

10. S. C. Davis et al., "Image-guided diffuse optical fluorescence tomography implemented with Laplacian-type regularization," Opt. Express 15(7), 4066-4082 (2007).

11. A. Godavarty et al., "Diagnostic imaging of breast cancer using fluorescence-enhanced optical tomography: phantom studies," J. Biomed. Opt. 9(3), 488-496 (2004).

12. N. Ducros et al., "Full-wavelet approach for fluorescence diffuse optical tomography with structured illumination," Opt. Lett. 35(21), 3676-3678 (2010).

13. J. Dutta et al., "Illumination pattern optimization for fluorescence tomography: theory and simulation studies," Phys. Med. Biol. 55(10), 2961-2982 (2010).

14. V. Ntziachristos et al., "Fluorescence molecular tomography resolves protease activity in vivo," Nat. Med. 8(7), 757-761 (2002).

15. N. Deliolanis et al., "Free-space fluorescence molecular tomography utilizing $360^{\circ}$ geometry projections," Opt. Lett. 32(4), 382-384 (2007).

16. C. Li et al., "A three-dimensional multispectral fluorescence optical tomography imaging system for small animals based on a conical mirror design," Opt. Express. 17(9), 7571-7585 (2009).

17. J. H. Lee et al., "Non-contact small animal fluorescence imaging system for simultaneous multi-directional angular-dependent data acquisition," Biomed. Opt. Exp. 5(7), 2301-2316 (2014).

18. D. Wang et al., "Noncontact full-angle fluorescence molecular tomography system based on rotary mirrors," Appl. Opt. 54(23), 7062-7070 (2015).

19. J. Dutta et al., "Joint $\mathrm{L}^{1}$ and total variation regularization for fluorescence molecular tomography," Phys. Med. Biol. 57(6), 1459-1476 (2012).

20. H. Yi et al., "Reconstruction algorithms based on 11-norm and 12-norm for two imaging models of fluorescence molecular tomography: a comparative study," J. Biomed. Opt. 18(5), 056013 (2013).

21. Y. An et al., "Meshless reconstruction method for fluorescence molecular tomography based on compactly supported radial basis function," J. Biomed. Opt. 20(10), 105003 (2015).

22. S. M. Hejazi, S. Sarkar, and Z. Darezereshki, "Fast multislice fluorescence molecular tomography using sparsity-inducing regularization," J. Biomed. Opt. 21(2), 026012 (2016).

23. D. Zhu and C. Li, "Accelerated image reconstruction in fluorescence molecular tomography using a nonuniform updating scheme with momentum and ordered subsets methods," J. Biomed. Opt. 21(1), 016004 (2016).

24. J. Zhang et al., "Iterative correction scheme based on discrete cosine transform and 11 regularization for fluorescence molecular tomography with background fluorescence," EEE Trans. Biomed. Eng. 63(6), 11071115 (2016).

25. Y. An et al., "A novel region reconstruction method for fluorescence molecular tomography," IEEE Trans. Biomed. Eng. 62(7), 18181826 (2015).

26. X. Zhang et al., "Shape-based reconstruction of dynamic fluorescent yield with a level set method," Biomed. Eng. Online 15(1), 6 (2016).

27. F. Leblond et al., "Pre-clinical whole-body fluorescence imaging: review of instruments, methods and applications," J. Photochem. Photobiol. B: Biol. 98(1), 77-94 (2010).

28. S. C. Davis et al., "Magnetic resonance-coupled fluorescence tomography scanner for molecular imaging of tissue," Rev. Sci. Instrum. 79(6), 064302 (2008).

29. R. B. Schulz et al., "Hybrid system for simultaneous fluorescence and X-ray computed tomography," IEEE Trans. Med. Imaging 29(2), 465473 (2010).

30. C. Li et al., "Three-dimensional fluorescence optical tomography in small animal imaging using simultaneous positron-emission-tomography priors," Opt. Lett. 34(19), 2933-2935 (2009).

31. S. C. Davis et al., "Comparing implementations of magnetic resonanceguided fluorescence molecular tomography for diagnostic classification of brain tumors," J. Biomed. Opt. 15(5), 051602 (2010). 
32. A. Ale et al., "Imaging performance of a hybrid x-ray computed tomography-fluorescence molecular tomography system using priors," Med. Phys. 37(5), 1976-1986 (2010).

33. F. Stuker et al., "Hybrid small animal imaging system combining magnetic resonance imaging with fluorescence tomography using single photon avalanche diode detectors," IEEE Trans. Med. Imaging 30(6), 1265-1273 (2011).

34. J. Peter et al., "Development and initial results of a tomographic dualmodality positron/optical small animal imager," IEEE Trans. Nucl. Sci. 54(5), 1553-1560 (2007).

35. Y. Lin et al., "Quantitative fluorescence tomography using a combined tri-modality FT/DOT/XCT system," Opt. Express 18(8), 7835-7850 (2010).

36. M. Liu et al., "In vivo pentamodal tomographic imaging for small animals," Biomed. Opt. Exp. 8(3), 1356-1371 (2017).

37. R. W. Holt, S. Davis, and B. W. Pogue, "Regularization functional semiautomated incorporation of anatomical prior information in imageguided fluorescence tomography," Opt. Lett. 38(14), 2407-2409 (2013).

38. G. Wang and J. Qi, "PET image reconstruction using kernel method," IEEE Trans. Med. Imaging 34(1), 61-71 (2015).

39. D. Zhu and C. Li, "Nonconvex regularizations in fluorescence molecular tomography for sparsity enhancement," Phys. Med. Biol. 59(12), 2901-2912 (2014).

40. D. Zhu et al., "Comparison of regularization methods in fluorescence molecular tomography," Photonics 1(2), 95-109 (2014).

41. S. R. Arridge and J. C. Hebden, "Optical imaging in medicine: II. Modelling and reconstruction," Phys. Med. Biol. 42(5), 841-853 (1997).

42. F. Fedele, J. Laible, and M. Eppstein, "Coupled complex adjoint sensitivities for frequency domain fluorescence tomography: theory and vectorized implementation," J. Comput. Phys. 187(2), 597-619 (2003).

43. D. Zhu and $\mathrm{C}$. $\mathrm{Li}$, "Nonuniform update for sparse target recovery in fluorescence molecular tomography accelerated by ordered subsets," Biomed. Opt. Exp. 5(12), 4249-4259 (2014).
44. B. Schölkopf, A. Smola, and K.-R. Müller, "Kernel principal component analysis," in Int. Conf. on Artificial Neural Networks, pp. 583-588, Springer (1997).

45. Y. Wang and L. Ying, "Undersampled dynamic magnetic resonance imaging using kernel principal component analysis," in 36th Annual Int. Conf. of the IEEE Engineering in Medicine and Biology Society, pp. 1533-1536, IEEE (2014).

46. A. Berlinet and C. Thomas-Agnan, Reproducing Kernel Hilbert Spaces in Probability and Statistics, Springer Science (2004).

47. F. Nouizi et al., "An accelerated photo-magnetic imaging reconstruction algorithm based on an analytical forward solution and a fast jacobian assembly method," Phys. Med. Biol. 61(20), 7448-7465 (2016).

48. T. Hofmann, B. Schölkopf, and A. J. Smola, "Kernel methods in machine learning," Ann. Stat. 36, 1171-1220 (2008).

49. Y. Zhao, R. Baikejiang, and C. Li, "Application of kernel method in fluorescence molecular tomography," Proc. SPIE 10057, 100570P (2017).

50. Y. Zhao et al., "Optimization and performance evaluation of a conical mirror based fluorescence molecular tomography imaging system," Proc. SPIE 9788, 97880R (2016).

51. Q. Fang and D. A. Boas, "Tetrahedral mesh generation from volumetric binary and grayscale images," in Proc. of IEEE Int. Symp. on Biomedical Imaging: From Nano to Macro, pp. 1142-1145, IEEE (2009).

52. Y. Zhao et al., "3D mouse shape reconstruction based on phase-shifting algorithm for fluorescence molecular tomography imaging system," Appl. Opt. 54(32), 9573-9582 (2015).

53. M. C. Lun, W. Zhang, and C. Li, "Sensitivity study of x-ray luminescence computed tomography," Appl. Opt. 56(11), 3010-3019 (2017).

Biographies for the authors are not available. 